\title{
GMRT observations of the field of INTEGRAL X-ray sources - I
}

\author{
M. Pandey ${ }^{1}$, R. K. Manchanda ${ }^{2}$, A. P. Rao ${ }^{3}$, P. Durouchoux ${ }^{4}$, and Ishwara-Chandra ${ }^{3}$ \\ 1 Department of Physics, Mumbai University, 400098 Mumbai, India \\ e-mail: mamta@ncra.tifr.res.in \\ 2 Department of Astronomy and Astrophysics, TIFR, Colaba, 400005 Mumbai, India \\ 3 NCRA, TIFR, Post Bag 3, Ganeshkhind, 411007 Pune, India \\ 4 CNRS FRE 2591/CEA Saclay, DSM/DAPNIA/SAP, 91191 Gif-sur-Yvette Cedex, France
}

Received 5 November 2004 / Accepted 8 September 2005

\begin{abstract}
Since its launch the INTEGRAL observatory has discovered a variety of hard X-ray sources in the Galactic plane. Using GMRT, we have made repeated observations of these sources to search for the radio counterparts of seventeen of them at low frequencies. The source positions were taken from the various ATEL and IAUC announcements reporting their discovery. Possible radio counterparts for seven of these sources, IGR J06074+2205, IGR J15479-4529, IGR J16479-4514, IGR J17091-3624, IGR J18027-1455, IGR J18539+0727 and IGR J21247+5058 were detected within $3 \sigma$ of the positional uncertainty derived from the INTEGRAL observations. The offset in the radio position was calculated using the positions mentioned in the ATEL. We have also analyzed the available NVSS images for some of these fields at $1.4 \mathrm{GHz}$ along with our observations. In this paper we present the radio images and the best fit positions for the positive detections. The X-ray variability for some of the sources within the time scales of $100 \mathrm{~s}$ to $1 \mathrm{ks}$ as seen in the RXTE/PCA light curves suggests their Galactic origin and possible binary nature. We discuss briefly the characteristics of these sources from the available information at different wave bands.
\end{abstract}

Key words. X-ray: binaries - binaries: close - radiation mechanism: nonthermal - instrumentation: high angular resolution instrumentation: interferometers

\section{Introduction}

Since its launch in Oct. 2002 the International Gamma Ray Astrophysics Laboratory, (INTEGRAL), has discovered a number of new hard X-ray/ soft $\gamma$-ray sources ${ }^{1}$ within the Galactic plain mainly towards the Galactic center region. These sources were discovered with the IBIS instrument, which has a large field of view of $29^{\circ} \times 29^{\circ}$ and a sensitivity up to $1 \mathrm{MeV}$ (Lebrun et al. 2003). Most of these new Galactic sources are soft gamma-ray emitters in the $20-100 \mathrm{keV}$ energy range and have been identified as X-ray binary systems (XRBs) (Bird et al. 2004).

The Galactic XRBs sources are accreting binary systems with a black hole (BH) or neutron star (NS) as the compact object. Apart from persistent X-ray emission from a number of these binaries, a large fraction of these systems are transient in nature, and brighten up sporadically with each source having some unique characteristics. Since observations of superluminal outflows during the transient burst of GRS 1915+105 (Mirabel \& Rodríguez 1994), a new subclass, characterized by the presence of jets in X-ray binaries, has been established.

1 See

http://isdc . unige.ch/ rodrigue/html/igrsources .html for an updated information on the list of all INTEGRAL sources.
The radio observations of some of these Galactic sources during quiescent and flaring modes using radio interferometers suggest that their morphology is similar to quasars, hence they are named microquasars. A compact core and two sided radio jets have been commonly observed in these sources during VLBI observations (Mirabel et al. 1999). In most of these sources the compact object is believed to be a black hole candidate (BHC); however, for Sco X-1, CI Cam and Cir X-1, the compact companion is determined to be a neutron star. Variability pattern in the radio light curve were seen for GRS 1915+105 (Mirabel et al. 1994, 1997, 1998). The X-ray emission from this source has also revealed a rich class of flux variability and temporal features (Belloni et al. 1997).

We have been carrying out a programme of monitoring of the new INTEGRAL sources since Aug. 2003 with the Giant Metrewave Radio Telescope, (GMRT) with a view:

1. To map the field of new X-ray sources at low frequencies in order to find the radio counterparts and provide the exact position coordinates with the arcsec resolution of GMRT at meter wavelengths.

2. To determine a possible association of the source by combining the radio, infrared and X-ray data and to establish the nature (low mass X-ray binary (LMXB) or high mass X-ray binary (HMXB)) of the source based on the 
magnitude of any infrared counterpart. Both these systems have a NS or $\mathrm{BH}$ as one of the component. In the case of HMXB the other component is a massive star, usually a Be star or a blue super-giant of $\sim 8-20 M_{\odot}$. For LMXBs the second component (donor) is a main sequence star. In some cases the donor can either be a degenerate or an evolved (sub-giant or red giant).

3. To distinguish between Galactic (compact) and extragalactic (extended) sources based on their statistically known radio morphology.

The radio data at different frequencies can help in unveiling the nature of these new sources and physics underlying the emission/absorption processes (Revnivtsev et al. 2003a).

In this paper we report the results obtained from radio observations performed on seventeen new sources with GMRT at $0.61 \mathrm{GHz}$ and $1.28 \mathrm{GHz}$. The selection criteria applied to detect possible radio-X-ray association discussed below are derived from our rigorous study of the known XRBs and $\mathrm{BH}$ jet sources. This was initiated in 2001 using GMRT (Pandey et al. 2004) to establish the long term spectral behavior of these sources at radio frequencies correlated to their X-ray properties.

The selection criteria for a true radio-X-ray association of the $\mathrm{X}$-ray sources is as follows:

1. The number of extragalactic sources with flux densities above $2.5 \mathrm{mJy}$ in the sky at $0.61 \mathrm{GHz}$ in $10^{\prime} \times 10^{\prime}$ area is typically $\sim 5$ whereas the number of sources expected above the flux density of $1.1 \mathrm{mJy}$ in $10^{\prime} \times 10^{\prime}$ area at $1.28 \mathrm{GHz}$ is typically $\sim 2$ (Condon et al. 1999). The number of sources decreases with: (i) the increase in flux density and (ii) decrease in the field area. The above source counts (number of sources) are used as typical limit for the extragalactic sources in the given area. The number of sources above this limit in the given area are considered as Galactic sources. The occurance of number of Galactic radio sources in the field of INTEGRAL source within the X-ray position error circle is statistically higher in number compared to extragalactic radio sources. Also this source count varies from the Galactic center to further away along the Galactic plane with lower probability in the latter region.

2. The X-ray binaries have compact radio morphology with the radio emission being non-thermal in origin. The variability in the radio flux density is a prominent feature in these sources. The radio spectra of microquasars show flattening or power law decay at high radio frequencies with radio emission coming from the jet, e.g. GRS 1915+105, SS433, Cyg X-1, and spectral turnover at low radio frequencies with radio emission coming from the radio lobe (Ishwara-Chandra et al. 2005). The radio emission is quenched when the source is in a high soft X-ray state. Also the radio emission from a few of these sources is synchrotron self absorbed below $1.28 \mathrm{GHz}$, e.g. X1812-121, XTE J1720-318, 1E1740.7-294 (Pandey et al. 2004). The $\mathrm{X}$-ray characteristics include prominent fluorescent emission from the iron line at $6.4 \mathrm{keV}$ and a hard X-ray tail showing rapid variability of the order of $\sim 10-100 \mathrm{~s}$. The infrared and optical counterparts are associated with the
X-ray binary source. The infrared emission originates from circumstellar disks undergoing major expansion coincident with phases of X-ray activity and the evaporation of the disk material due to heating gives rise to optical emission. Thus a compact radio source within the $3 \sigma$ X-ray position uncertainty limit satisfying the above criteria is very likely to be associated with the Galactic X-ray source.

3. The radio sources with extended radio morphology and with their radio emission being non-thermal in origin have a spectral index, $\alpha \sim-0.7\left(S_{v}=v^{\alpha}\right)$, are classified as extragalactic radio sources. Their radio emission is mostly nonvariable. The $\mathrm{X}$-ray light curve shows variability on larger timescales; however, variability of the order of $\sim 100 \mathrm{~s}$ is reported for few extragalactic sources. Thus a radio source within the $3 \sigma \mathrm{X}$-ray position uncertainty limit satisfying the above criteria is most likely not associated with the Galactic $\mathrm{X}$-ray source.

The observations of these seventeen sources presented here were made in the Aug. 2003 observation cycle with GMRT. Further observations of 23 sources belonging to this class were conducted in Aug. 2004 and the data analysis is in progress. The target sources are those discovered during the INTEGRAL observation scans on the Galactic plane, and during guest observations and Crab calibrations; they are listed in Table 1.

\section{Observations and analysis}

The radio observations were carried out at $0.61 \mathrm{GHz}$ and $1.28 \mathrm{GHz}$ with a bandwidth of $16 / 32 \mathrm{MHz}$ using the GMRT (Swarup 1991). The half power beam width for the GMRT antenna at $0.61 \mathrm{GHz}$ is $\sim 43^{\prime}$ and at $1.28 \mathrm{GHz}$ is $\sim 26^{\prime}$. The measured position offset of the possible GMRT radio counterparts with respect to the $\mathrm{X}$-ray sources is below $\sim 5^{\prime}$, hence primary beam corrections do not contribute a significant change to the images. The gain decreases significantly in the field at low radio frequencies, i.e. $0.61 \mathrm{GHz}$, hence gain depression corrections or system temperature corrections were applied to the flux densities of the radio counterparts. The flux density scale was set by observing the primary calibrators 3C 286, 3C 147 and 3C 48. Phase calibrators were observed near the target source for $\sim 5$ min scans interleaved with 25 min scans on the INTEGRAL sources. The sample time was $16 \mathrm{~s}$. The data recorded from GMRT were converted into FITS files and analyzed using the Astronomical Image Processing System (AIPS). A self calibration on the data was performed to correct for phase related errors and improve the image quality. $7-10 \%$ of the data was affected by interference and was carefully removed. Ionospheric scintillation effects were not seen in the 0.61 and $1.28 \mathrm{GHz}$ data during our observations. Possible radio counterparts were detected for seven out of seventeen sources and for other sources no possible radio counterpart was detected within the $3 \sigma$ error box of the X-ray source. Table 2 summarizes our results for the new sources along with best-fit radio positions for the counterpart and the position offsets of this counterpart with respect to the X-ray positions. Column 4 gives the peak and total flux density for the point and extended source respectively. The rms noise 
Table 1. List of target INTEGRAL sources observed with GMRT.

\begin{tabular}{|c|c|c|c|c|c|c|}
\hline Source & Type & $\begin{array}{l}\text { Integral } \\
\text { Pos. Unc } \\
1.6 \sigma\end{array}$ & $\begin{array}{l}\text { Variable } \\
100 \mathrm{~s}-1 \mathrm{ks} \\
\text { IBIS/ISGRI }\end{array}$ & $\begin{array}{l}\text { X-ray } \\
\text { Flux } \\
\text { (mCrab) } \\
15-40 \mathrm{keV}\end{array}$ & $\begin{array}{l}\text { X-ray } \\
\text { State } \\
\text { during } \\
\text { GMRT obs. }\end{array}$ & $\begin{array}{l}\text { GMRT } \\
\text { detection } \\
\text { within X-ray } \\
3 \sigma \text { error box }\end{array}$ \\
\hline IGR J06074+2205 & - & $2^{\prime}$ & Yes & 15 & high soft & Yes \\
\hline IGR J15479-4529 & - & $2^{\prime}$ & Yes & 2 & - & Yes \\
\hline IGR J16316-4028 & - & $3^{\prime}$ & Yes & 25 & - & Yes \\
\hline IGR J16318-4848 & $\operatorname{sgB}(e)(H M X B)$ & $4^{\prime \prime}$ & Yes & $50-100$ & high soft & No \\
\hline IGR J16320-4751 & $\operatorname{HMXB}(?)$ & $4^{\prime \prime}$ & Yes & $10-50$ & - & No \\
\hline IGR J16418-4532 & - & $2^{\prime}$ & - & 2 & - & No \\
\hline IGR J16479-4514 & & $3^{\prime}$ & Yes & 12 & high soft & Yes \\
\hline IGR J17091-3624 & $\mathrm{XB}^{1}(\mathrm{BHC})$ & $0.8^{\prime}$ & Yes & 40 & low hard & Yes \\
\hline & ? & & & - & - & - \\
\hline IGR J17391-3021 & $\begin{array}{l}\mathrm{Be} /(\mathrm{NS}) \mathrm{HMXB}^{2} \\
?\end{array}$ & $\begin{array}{l}1^{\prime \prime} \\
\text { Chandra }\end{array}$ & Yes & $\begin{array}{l}150 \\
-\end{array}$ & - & No \\
\hline IGR J17544-2619 & $\operatorname{HMXB}(?)$ & $4^{\prime \prime}$ & Yes & 60 & high soft & No \\
\hline IGR J17597-2201 & Neutron Star, LMXB & $2^{\prime}$ & Yes & 5 & - & No \\
\hline IGR J18027-1455 & Extragalactic $^{3}$ & $2^{\prime}$ & Yes & - & high soft & Yes \\
\hline IGR J18325-0756 & - & $2-3^{\prime}$ & Yes & - & - & No \\
\hline IGR J18483-0311 & - & $2^{\prime}$ & Yes & 10 & - & No \\
\hline IGR J18539+0727 & $\begin{array}{l}\mathrm{XB}^{4} \\
(\mathrm{BHC})\end{array}$ & $3^{\prime}$ & Yes & $\begin{array}{l}20 \\
-\end{array}$ & low hard & Yes \\
\hline IGR J19140+0951 & $\begin{array}{l}\mathrm{HMXB}^{5} \\
(\mathrm{NS})\end{array}$ & $1.3^{\prime}$ & Yes & $\begin{array}{l}50--100 \\
-\end{array}$ & high soft & No \\
\hline IGR J21247+5058 & Radio Galaxy & $2^{\prime}$ & Yes & - & low hard & Yes \\
\hline
\end{tabular}

${ }^{1}$ Revnivtsev et al. (2003a), ${ }^{2}$ Smith et al. (2003), ${ }^{3}$ Masetti et al. (2004), ${ }^{4}$ Revnivtsev et al. (2003b), ${ }^{5}$ Rodriguez et al. (2005).

given in Col. 5 of Table 2 corresponds to the average background noise in the image field and is higher in the Galactic plane. In the radio images presented below, the boxes show the known field sources (X-ray, gamma-ray, optical, infrared, radio) from the files available from the NASA extragalactic Database (NED), NVSS (Condon et al. 1998), 2MASS and Digital Sky Survey (DSS). The number of infrared and optical sources expected within a $10^{\prime}$ area in the Galactic region was derived from the image obtained from FITS files available from the 2MASS and DSS surveys at: http:// skys.gsfc.nasa.gov/cgi-bin/skvadvanced.pl. The expected number of infrared and optical sources are $\sim 6$ and $\sim 1.2$ respectively; however, in the radio images shown in this paper, only the sources in close proximity to the radio source have been marked. We also discuss briefly the spectral properties in the radio band (non-simultaneous radio observations) of the radio counterparts for which other data are available. The bold circle marked with "A" indicates a possible GMRT radio counterpart close to the $\mathrm{X}$-ray source. We have grouped sources with a genuine radio-X-ray association, no association and no radio information, in Sect. 3.

\section{Results}

Imaging at radio wavelengths are is important because interferometry can provide precise sub-arcsec position measurements, thus improving the error boxes obtained from X-ray data.
In addition, interstellar extinction has very little effect at low radio wavelengths.

\subsection{X-ray sources with a radio source in the field and radio- $X$-ray association}

IGR J17091-3624: this microquasar was discovered at a flux density level of $\sim 20 \mathrm{mCrab}$ between $40-100 \mathrm{keV}$ (Kuulkers et al. 2003). BeppoSAX detected the source at a flux density level of $14-20 \mathrm{mCrab}$ in the $2-10 \mathrm{keV}$ band, suggesting a very steep photon spectrum consistent with a spectral index of $3.0 \pm 0.4$ or $k T=4.3 \pm 1.4 \mathrm{keV}$ from the thermal bremsstrahlung (Zand et al. 2003). During a 3 ks observation of the source with RXTE/PCA in the 2-20 keV band, the observed flux density was $\sim 4 \mathrm{mCrab}$. The $\mathrm{X}$-ray light curve of the source shows a large variability on time scales from several tenths to several tens of seconds. The energy spectrum of the source was fitted by a power law index of $1.43 \pm 0.03$. No visible absorption was detected at the lower energies (Revnivtsev \& Lutovinov 2003). The combined X-ray data from BeppoSAX and INTEGRAL also suggest a variable nature of the source with flare episodes in Apr. 2003 and Sep. 2003 (Kuulkers et al. 2003). The radio emission from the source region was detected during observations with the Very Large Array (VLA) at $4.9 \mathrm{GHz}$ with a flux density of $1.8 \pm 0.3 \mathrm{mJy}$ and they showed significant variability in flux density (Rupen et al. 2003a).

In our observation with GMRT, a radio source was detected within the $1.6 \sigma$ position uncertainty of the X-ray sources at a 
Table 2. Possible radio counterparts of target INTEGRAL sources observed with GMRT.

\begin{tabular}{|c|c|c|c|c|c|c|c|c|}
\hline Source & $\begin{array}{l}\text { Date } \\
\mathrm{dd} / \mathrm{mm} / \mathrm{yy}\end{array}$ & $\begin{array}{l}v \\
(\mathrm{GHz})\end{array}$ & $\begin{array}{l}S_{v} \\
(\mathrm{mJy})\end{array}$ & $\begin{array}{l}\sigma \\
\left(\mathrm{mJy} \mathrm{b}^{-1}\right)\end{array}$ & $\begin{array}{l}\text { Radio } \\
\text { Pos. GMRT } \\
\text { RA and Dec }\end{array}$ & $\begin{array}{l}\text { Pos. Off. } \\
\text { w.r.t } \\
\text { Integral } \\
\text { Pos. }\end{array}$ & $\begin{array}{l}\text { Radio } \\
\text { Struc. } \\
\text { GMRT }\end{array}$ & $\begin{array}{l}S_{v} \\
\text { (Total) } \\
\text { NVSS } \\
1.4 \mathrm{GHz} \\
(\mathrm{mJy})\end{array}$ \\
\hline \multirow[t]{4}{*}{ IGR J06074+2205 } & $01 / 02 / 04$ & 1.28 & $36.00 \pm 1.12$ & 0.64 & $\begin{array}{l}\text { 06h } 07 \mathrm{~m} \mathrm{18.45s} \pm 0.005 \\
22 \mathrm{~d} 04^{\prime} 52.49^{\prime \prime} \pm 0.03\end{array}$ & $1.29^{\prime}$ & Point & 36 \\
\hline & $09 / 04 / 04$ & 0.61 & $78.65 \pm 1.29$ & 1.33 & & & & \\
\hline & $12 / 04 / 04$ & 0.61 & $83.49 \pm 1.30$ & 0.65 & & & & \\
\hline & $02 / 05 / 04$ & 0.61 & $79.86 \pm 1.42$ & 1.57 & & & & \\
\hline IGR J15479-4529 & $02 / 02 / 04$ & 1.28 & 22.2 & 0.5 & $\begin{array}{l}15 \mathrm{~h} 47 \mathrm{~m} 44.26 \mathrm{~s} \pm 0.105 \\
-45 \mathrm{~d} 32^{\prime} 35.22^{\prime \prime} \pm 1.76\end{array}$ & $3.98^{\prime}$ & Extended & N/A \\
\hline \multirow[t]{3}{*}{ IGR J16316-4028 } & $02 / 02 / 04$ & 1.28 & $\leq 0.8$ & 0.19 & & & & \\
\hline & 09/04/04 & 0.61 & $\leq 6.00$ & 1.35 & & & & \\
\hline & $12 / 04 / 04$ & 0.61 & $\leq 4.80$ & 1.18 & & & & \\
\hline \multirow[t]{3}{*}{ IGR J16318-4848 } & 04/08/03 & 0.61 & $\leq 6.50$ & 2.13 & & & & N/A \\
\hline & $15 / 09 / 03$ & 0.61 & $\leq 6.60$ & 2.20 & & & & \\
\hline & 09/04/04 & 0.61 & $\leq 7.00$ & 2.41 & & & & \\
\hline \multirow[t]{3}{*}{ IGR J16320-4751 } & $29 / 07 / 03$ & 1.28 & $\leq 3.00$ & 0.83 & & & & $\mathrm{~N} / \mathrm{A}$ \\
\hline & $04 / 08 / 03$ & 0.61 & $\leq 4.00$ & 1.23 & & & & \\
\hline & $15 / 09 / 03$ & 0.61 & $\leq 4.00$ & 1.04 & & & & \\
\hline IGR J16418-4532 & $02 / 02 / 04$ & 1.28 & $\leq 1.00$ & 0.33 & & & & $\mathrm{~N} / \mathrm{A}$ \\
\hline IGR J16479-4514 & $02 / 02 / 04$ & 1.28 & 2445.45 & 1.24 & $\begin{array}{l}16 \mathrm{~h} 47 \mathrm{~m} 47.75 \mathrm{~s} \pm 0.153 \\
-45 \mathrm{~d} 17^{\prime} 07^{\prime \prime} \pm 1.97\end{array}$ & $3.30^{\prime}$ & Extended & N/A \\
\hline \multirow[t]{2}{*}{ IGR J17091-3624 } & $16 / 09 / 03$ & 0.61 & $2.50 \pm 0.73$ & 0.60 & $\begin{array}{l}17 \mathrm{~h} 09 \mathrm{~m} 02 \mathrm{~s} \pm 0.191 \\
-36 \mathrm{~d} 23^{\prime} 33^{\prime \prime} \pm 2.84\end{array}$ & $0.99^{\prime}$ & Point & 1.5 \\
\hline & $02 / 02 / 04$ & 1.28 & $1.10 \pm 0.16$ & 0.19 & & & & \\
\hline IGR J17391-3021 & $02 / 02 / 04$ & 1.28 & $\leq 1.04$ & 0.19 & & & & $\leq 2.5$ \\
\hline IGR J17544-2619 & $12 / 04 / 04$ & 0.61 & $\leq 7.35$ & 2.46 & & & & $\leq 3$ \\
\hline IGR J17597-2201 & $16 / 09 / 03$ & 0.61 & $\leq 7.90$ & 2.42 & & & & $\leq 3.5$ \\
\hline IGR J18027-1455 & $02 / 05 / 04$ & 0.61 & $10.72 \pm 2.25$ & 2.32 & $\begin{array}{l}18 \mathrm{~h} 02 \mathrm{~m} 42.75 \mathrm{~s} \pm 0.134 \\
-14 \mathrm{~d} 50^{\prime} 49.21^{\prime \prime} \pm 3.93\end{array}$ & $4.18^{\prime}$ & Point & 12.3 \\
\hline IGR J18325-0756 & $16 / 09 / 03$ & 0.61 & $\leq 3.00$ & 0.75 & & & & $\leq 3.5$ \\
\hline \multirow[t]{2}{*}{ IGR J18483-0311 } & $16 / 09 / 03$ & 0.61 & $\leq 4.24$ & 1.27 & & & & \\
\hline & $02 / 02 / 04$ & 1.28 & $\leq 1.00$ & 0.15 & & & & $\leq 1.6$ \\
\hline \multirow[t]{2}{*}{ IGR J18539+0727 } & $02 / 02 / 04$ & 1.28 & $5.25 \pm 0.25 \pm 0.14$ & 0.16 & $\begin{array}{l}18 \mathrm{~h} 53 \mathrm{~m} 52.54 \mathrm{~s} \pm 0.028 \\
+07 \mathrm{~d} 30^{\prime} 39.07^{\prime \prime} \pm 0.43\end{array}$ & $3.64^{\prime}$ & $\begin{array}{l}\text { Double } \\
\text { Point }\end{array}$ & \\
\hline & & & $5.85 \pm 0.50$ & & $\begin{array}{l}18 \mathrm{~h} 53 \mathrm{~m} 52.19 \mathrm{~s} \pm 0.023 \\
+07 \mathrm{~d} 30^{\prime} 37.31^{\prime \prime} \pm 0.32\end{array}$ & & & 6 \\
\hline \multirow[t]{10}{*}{ IGR J19140+0951 } & $21 / 06 / 03$ & 0.61 & $\leq 5.50$ & 1.28 & & & & $\leq 1.6$ \\
\hline & $29 / 07 / 03$ & 1.28 & $\leq 0.80$ & 0.15 & & & & \\
\hline & $04 / 08 / 03$ & 0.61 & $\leq 5.50$ & 1.23 & & & & \\
\hline & $09 / 09 / 03$ & 0.61 & $\leq 5.00$ & 1.14 & & & & \\
\hline & $15 / 09 / 03$ & 0.61 & $\leq 5.70$ & 1.90 & & & & \\
\hline & $02 / 02 / 04$ & 1.28 & $\leq 0.50$ & 0.15 & & & & \\
\hline & $07 / 04 / 04$ & 0.61 & $\leq 5.00$ & 1.43 & & & & \\
\hline & $12 / 04 / 04$ & 0.61 & $\leq 5.00$ & 1.28 & & & & \\
\hline & $02 / 05 / 04$ & 0.61 & $\leq 5.00$ & 1.64 & & & & \\
\hline & 03/05/04 & 0.61 & $\leq 5.00$ & 1.20 & & & & \\
\hline \multirow[t]{4}{*}{ IGR J21247+5058 } & $07 / 04 / 04$ & 0.61 & $184.81 \pm 1.59$ & 1.25 & $\begin{array}{l}21 \mathrm{~h} 24 \mathrm{~m} 39.63 \mathrm{~s} \pm 0.004 \\
50 \mathrm{~d} 58^{\prime} 25.55^{\prime \prime} \pm 0.04\end{array}$ & $0.57^{\prime}$ & Extended & 237 \\
\hline & $09 / 04 / 04$ & 0.61 & $178.41 \pm 1.38$ & 1.61 & & & & \\
\hline & $12 / 04 / 04$ & 0.61 & $173.81 \pm 1.59$ & 1.71 & & & & \\
\hline & $02 / 05 / 04$ & 0.61 & $177.71 \pm 1.17$ & 1.52 & & & & \\
\hline
\end{tabular}




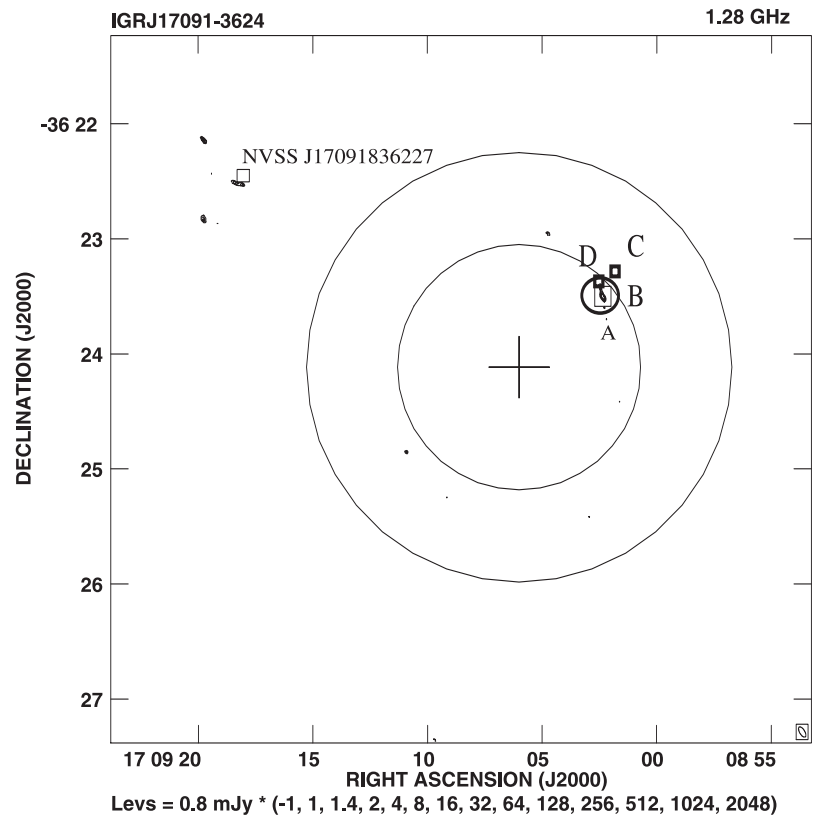

Fig. 1. GMRT image of IGR J17091-3624 at $1.28 \mathrm{GHz}$ with the INTEGRAL position marked with + . The small and large circles shows the INTEGRAL uncertainty error circles of $1.6 \sigma$ and $3 \sigma$. The bold circle marked with "A" show the radio source detected by GMRT. The boxes show the known field sources, B: NVSS 17092-3624, C: DSS J170901-3623 and D: 2MASS J170902-3623.

radio flux density of $1.10 \pm 0.16 \mathrm{mJy}$ at $1.28 \mathrm{GHz}$, at a $\mathrm{J} 2000$ position of RA: $17 \mathrm{~h} 09 \mathrm{~m} 02.3 \mathrm{~s}$ and Dec: $-36 \mathrm{~d} \mathrm{23} 3^{\prime} 33^{\prime \prime}$. The rms noise was $0.19 \mathrm{mJy} \mathrm{b}^{-1}$. The flux density at $0.61 \mathrm{GHz}$ was measured to be $2.50 \pm 0.73 \mathrm{mJy}$.

The GMRT image of the field is shown in Fig. 1. The source morphology is consistent with a point source. The compact nature of the radio source is also inferred from the Gaussian fit to the source flux density using the AIPS routine "JMFIT". In our analysis of the NVSS FITS files, we have detected a weak radio source with a flux density of $\sim 1.50 \pm 0.25 \mathrm{mJy}$ at the same position. The radio source marked "A" in the figure lies within the INTEGRAL position error circle of the source and is the most likely radio counterpart of the X-ray source. The GMRT (A) and NVSS (B) radio counterparts are coincident in position. The infrared source, (D), and the optical source, $(\mathrm{C})$, coincide in position with the radio source. Thus we confirm that the sources (B), (C) and (D) are associated with the X-ray binary source. Thus the infrared emission may come from a circumstellar disk undergoing major expansion coincident with phases of $\mathrm{X}$-ray activity and the optical emissions are due to heating and evaporation of the disk material. In order to enhance the significance of the possible association of the GMRT source with the X-ray object, we have plotted the radio flux density measurements of the source region at GMRT frequencies of 0.61 and $1.28 \mathrm{GHz}$ in Fig. 2. The observed flux density values for the NVSS source at $1.4 \mathrm{GHz}$ and the VLA flux density value at $4.9 \mathrm{GHz}$ are also plotted in the figure for reference. However, the observations are non-simultaneous. The spectral fit to the GMRT data corresponds to the falling power law of the form $\left(S \propto v^{\alpha}\right)$ with $\alpha=-0.62$. This is consistent with

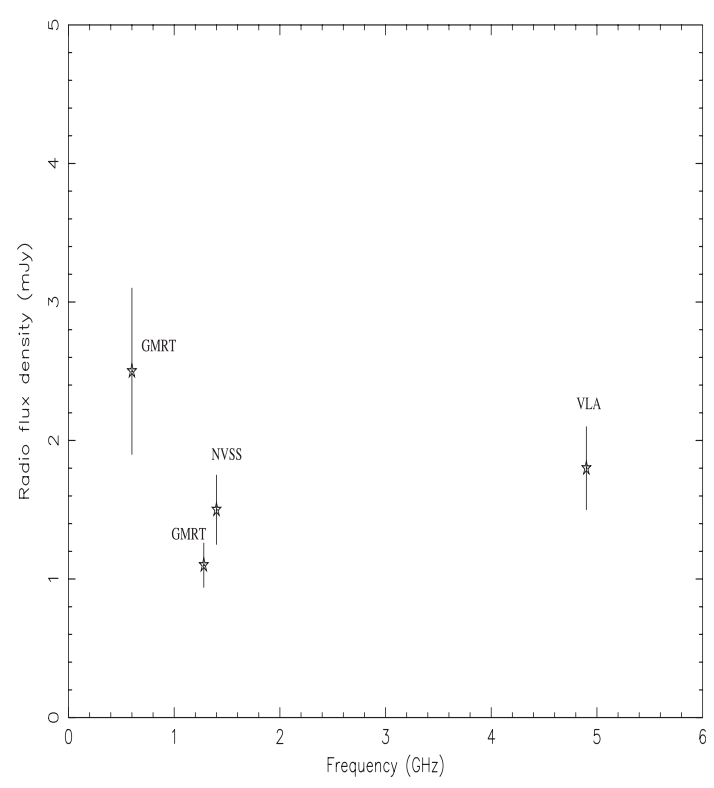

Fig. 2. Radio spectrum for IGR J17091-3624.

the radio emission from the other X-ray binary sources. If the high frequency observations are associated with the same emission region, the radio spectral fit will require a clear inflection to positive slope with $\alpha=+2.73$. This suggests a composite of two separate radio emitting regions for the source.

IGR J18027-1455: this source is an AGN candidate located in the Norma arm region (Walter et al. 2004; Masetti et al. 2004). A faint ROSAT X-ray source 1RXS J180245.5-145432 (Voges et al. 2000) lies well within the IBIS/ISGRI error circle. Two weak radio sources were discovered in the NVSS field at $1.4 \mathrm{GHz}$ (Combi et al. 2004). The first source, NVSS J180239-145453, located at J2000.0 ICRS position of RA: $18 \mathrm{~h} 02 \mathrm{~m} 39.94 \mathrm{~s}( \pm 0.25 \mathrm{~s})$, Dec: $-14 \mathrm{~d} \mathrm{54}$ $53.6^{\prime \prime}\left( \pm 3.3^{\prime \prime}\right)$ and 0.51 arcmin away from the X-ray source has a flux density of $6.9 \mathrm{mJy}$. The 2MASS image of the field gives an infrared source, 2MASS J180239-145453, coincident in position with the radio source NVSS J180239-145453. The second source, NVSS J180247-145451, at J2000.0 ICRS position of RA: $18 \mathrm{~h} 02 \mathrm{~m} 47.37 \mathrm{~s}( \pm 0.13 \mathrm{~s})$, Dec: $-14 \mathrm{~d} 54^{\prime} 51.6^{\prime \prime}\left( \pm 2.2^{\prime \prime}\right)$ is located 0.28 arcmin away from the X-ray position and has a flux density of $10.5 \mathrm{mJy}$. The field is further complicated by the presence of an extended IR source, 2MASXi J1802473-145454, with coordinates RA: 18h $02 \mathrm{~m} 47.3 \mathrm{~s}$, Dec: $-14 \mathrm{~d} 54^{\prime} 55^{\prime \prime}$ and magnitudes $J=13.18$, $H=12.02, K=10.94$, within the $2 \sigma$ position error ellipse of the second radio source. The average optical magnitudes of this IR source are $B=19.3, R=14.9$ and $I=13.8$ in the USNO-B1.0 catalog (Combi et al. 2005). The source has an optical counterpart DSS J180241-145453. The sources NVSS J180247-145451, 2MASXi J1802473-145454, DSS J180241-145453 and 1RXS J180245.5-145432 are therefore likely to be associated with the counterparts of IGR J18027-1455 (Combi et al. 2005). The GMRT observations were made at $0.61 \mathrm{GHz}$ and a image of the field is shown in Fig. 3. It is seen from the figure that GMRT 


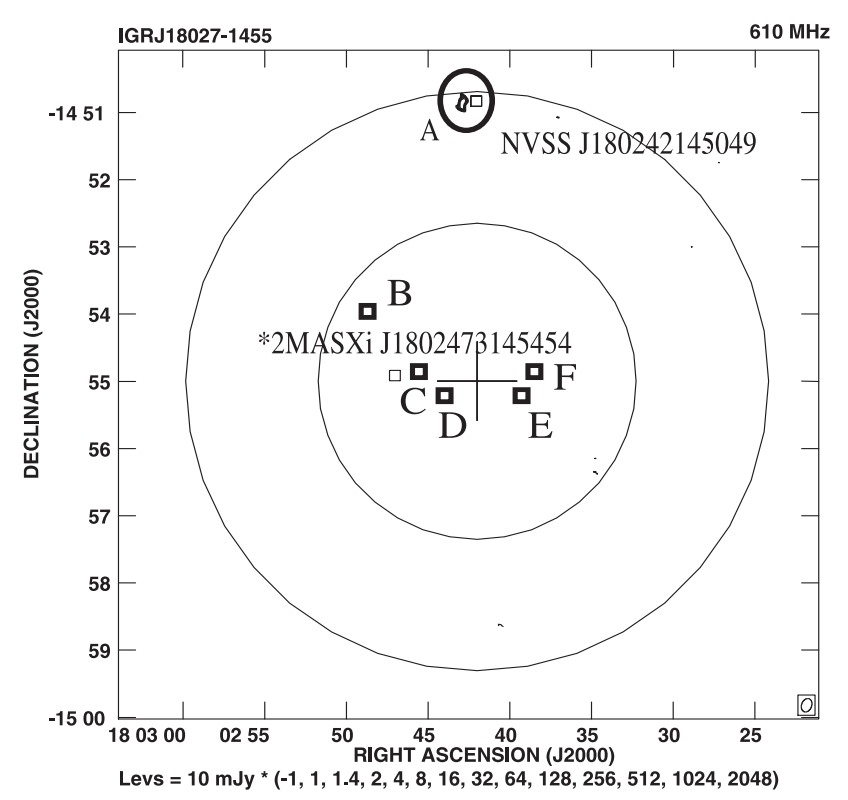

Fig. 3. GMRT image of IGR J18027-1455 at $0.61 \mathrm{GHz}$ with the INTEGRAL position marked with + . The small and large circles shows the INTEGRAL uncertainty error circles of $1.6 \sigma$ and $3 \sigma$. The bold circle marked with "A" shows a radio source detected by GMRT. The boxes show known field sources, B: 1RXS J180245.5-145432, C: NVSS J180247-145451, D: DSS J180241-145453, E: NVSS J180239-145453.6 and F: 2MASS J180239-145453.

data did not yield any significant flux from the directions of NVSS J180239-145453 (E), NVSS J180247-145451 (C), 2MASS source $(F)$ or the ROSAT (B) source position. The only point radio source seen in the GMRT data, (A), was at a flux density of $10.72 \pm 2.25 \mathrm{mJy}$ and is coincident with the NVSS source J180242-145049. The GMRT position for the radio source is RA: $18 \mathrm{~h} 02 \mathrm{~m} 42.75 \mathrm{~s}$ and Dec: $-14 \mathrm{~d} 50^{\prime} 49.21^{\prime \prime}$ with position offset $4.18^{\prime}$ with respect to the INTEGRAL position. The rms noise was $2.3 \mathrm{mJy} \mathrm{b}^{-1}$ and the NVSS flux density for the source is $12.3 \mathrm{mJy}$. With the detection of a higher flux density at $0.61 \mathrm{GHz}$ compared with NVSS data, we can infer that NVSS J180242-145049 is a flat spectrum source in the $0.61 \mathrm{GHz}$ to $1.4 \mathrm{GHz}$ range, assuming that the source is persistent in nature.

Non- detection of the 2 radio sources closest to the INTEGRAL X-ray source position during our observation, suggests that the radio sources show spectral turnover at low frequencies or are variable in nature. However in both the cases, a non-thermal origin of the radio emission is clearly favoured. Thus considering the NVSS flux density at $1.4 \mathrm{GHz}$ for the source NVSS J180247-145451 and the upper limit of $<7 \mathrm{mJy}$ from GMRT observations at $0.6 \mathrm{GHz}$, the spectral index derived is $\alpha=0.48$. The RXTE/ASM light curve for the hardness ratio of the X-ray source suggests that it was in a high soft state during our observations. Thus, NVSS J180247-145451 (C) is a possible radio counterpart of the X-ray source. The non-detection of NVSS J180239-145453 (E) and NVSS J180247-145451 (C) with GMRT in May 2004 and a clear detection in the
NVSS survey is consistent with the binary nature of the X-ray source.

However observation of IGR J18027-1455 region with the GMRT (2nd Mar. 2005) showed an evolution of the previous trends followed by the source in the radio regime. We have detected radio emission at $0.61 \mathrm{GHz}$ in the direction of the INTEGRAL source coincident in position with the NVSS (E) and $(\mathrm{C})$ sources; see Fig. 3. The radio morphology reveals the extended nature of the sources. The (E) and (C) sources have GMRT flux densities of $9.00 \pm 0.33$ and $5.00 \pm 0.35 \mathrm{mJy}$, respectively. A clear low frequency radio variability is established for the source by our observations. The ASM light curve for the source shows a flaring episode during MJD 53 350-53 360 i.e. 1st-11th Dec. 2004. This enhances the significance of the radio-X-ray association.

IGR J18539+0727: this source was discovered with the IBIS/ISGRI detector in Apr. 2003 (Lutovinov et al. 2003a). With the measured photon fluxes of $20 \mathrm{mCrab}$ between $15-40 \mathrm{keV}$ and $20 \mathrm{mCrab}$ between $40-100 \mathrm{keV}$, IGR J18539+0727 has the most unusual flat spectrum among the known X-ray sources in hard X-ray band. In a later observation of the source with RXTE/PCA, an average flux density level of $\sim 6 \mathrm{mCrab}$ was found with large variability on time scales from milli-seconds to seconds, but without any prominent features associated with quasi-periodic oscillations (QPO). The energy spectrum of the source fits a power law with spectral index $\sim-1.5$, and has appreciable low energy attenuation due to neutral hydrogen of $n_{\mathrm{H}} \sim(1.5 \pm 0.4) \times 10^{22}$ and a fluorescent iron emission line at $6.4 \mathrm{keV}$. The observed spectral and temporal characteristics of the X-ray source fits well with the standard model of AGNs (Ceballos et al. 2004).

During GMRT observations of the field, a fully resolved double point source with a flux density of $\sim 5.25 \pm 0.25 \mathrm{mJy}$ and $\sim 5.85 \pm 0.50 \mathrm{mJy}$ was detected from the J2000 position of, RA:

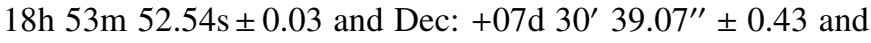
RA: $18 \mathrm{~h} 53 \mathrm{~m} 52.19 \mathrm{~s} \pm 0.023$ and Dec: $+07 \mathrm{~d} 30^{\prime} 37.31^{\prime \prime} \pm 0.32$, respectively, which lies within the $1.6 \sigma$ position error circle of $3^{\prime}$ of the X-ray source. The radio morphology resembles IGR J18027-1455 or an AGN. The computed position offset from the field center is $3.82^{\prime}$ and $3.64^{\prime}$ respectively as shown in Fig. 4. The GMRT source is coincident with the NVSS unresolved radio source in the field, for which the flux density is estimated as $\sim 6 \mathrm{mJy}$ at $1.4 \mathrm{GHz}$. The radio flux density measurements, although done at different epochs are consistent with a flat spectrum source between 1.28 and $1.4 \mathrm{GHz}$ and its persistent nature. Figure 4 also shows the 2MASS J18535+0731 (infrared, E) and DSS J18535+07315 (optical, C) are coincident in position with the radio source, thus suggesting a possible association with the radio source. A power law X-ray spectrum with strong photoabsorption at low energies and fluorescent iron emission line at $6.4 \mathrm{keV}$ clearly points to a binary nature for IGR J18539+0727, in which the emission may arise due to the reflection of the hard X-radiation of inner accretion-flow regions from an optically thick, cold accretion disk. Furthermore, the power density spectrum of the source shows a cutoff below a frequency of $\sim 0.01 \mathrm{~Hz}$, which places it in the category of a compact companion to a BHC (Van der Klis et al. 1999). The 


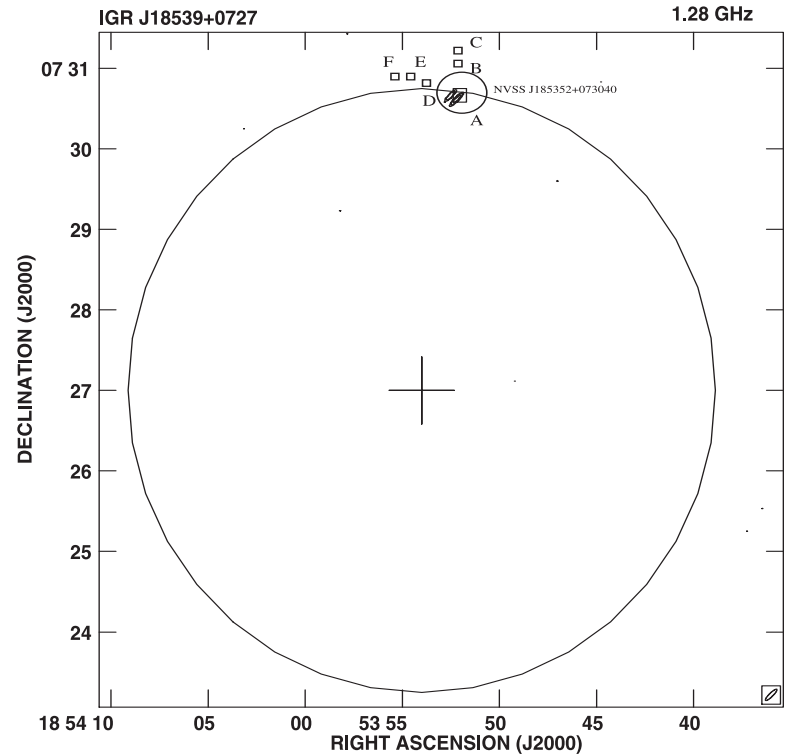

Levs $=0.7 \mathrm{mJy}^{*}(-3,1,1.400,2,2.800,4.200,8,16,32,64,128,256,512,1024)$

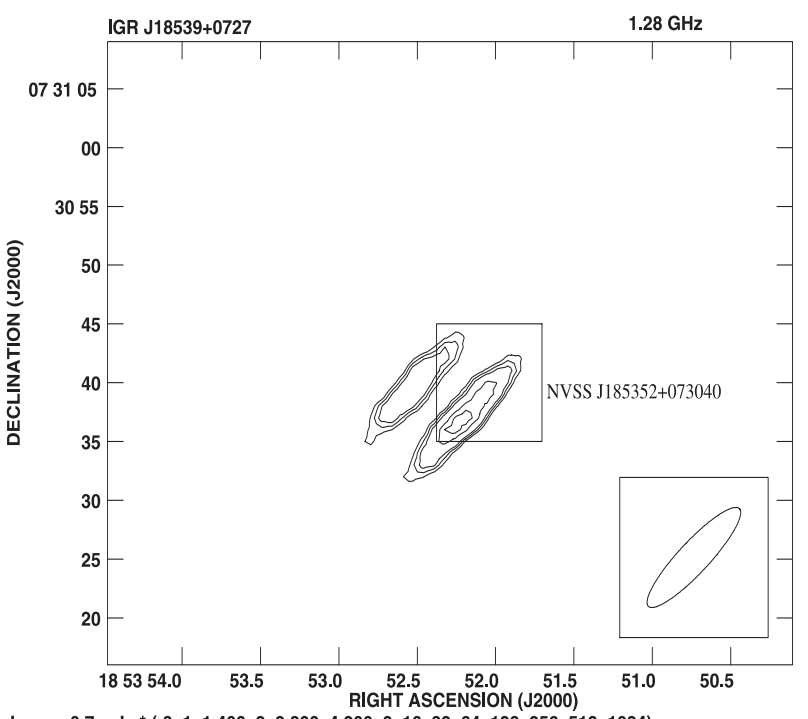

Levs $=0.7 \mathrm{mJy}{ }^{*}(-3,1,1.400,2,2.800,4.200,8,16,32,64,128,256,512,1024)$

Fig. 4. Top the GMRT image of IGR J18539+0727 at $1.28 \mathrm{GHz}$ with the INTEGRAL position marked with + . The circle shows the INTEGRAL uncertainty error circle of $1.6 \sigma$. The bold circle marked with "A" shows the radio source detected by GMRT. Below the blown up image of GMRT radio counterpart. The boxes show the known field sources, B: 2MASS J18535+0731, C: DSS J18535+07315, D: 2MASS J185352+07305, E: 2MASS J185353+07305 and F: DSS J185353+07305

presence of infrared and optical counterparts also supports the disk-jet morphology. Thus the hard X-ray source is associated with the radio counterpart detected by GMRT and we conclude from our result that IGR J18539+0727 is an AGN.

\section{2. $X$-ray sources with a radio source in the field and no radio- $X$-ray association}

IGR J06074+2205: the transient X-ray source IGR J06074+2205 was detected with a flux density of $\sim 7 \mathrm{mCrab}$ between 3-10 keV by INTEGRAL (Chenevez et al. 2004).

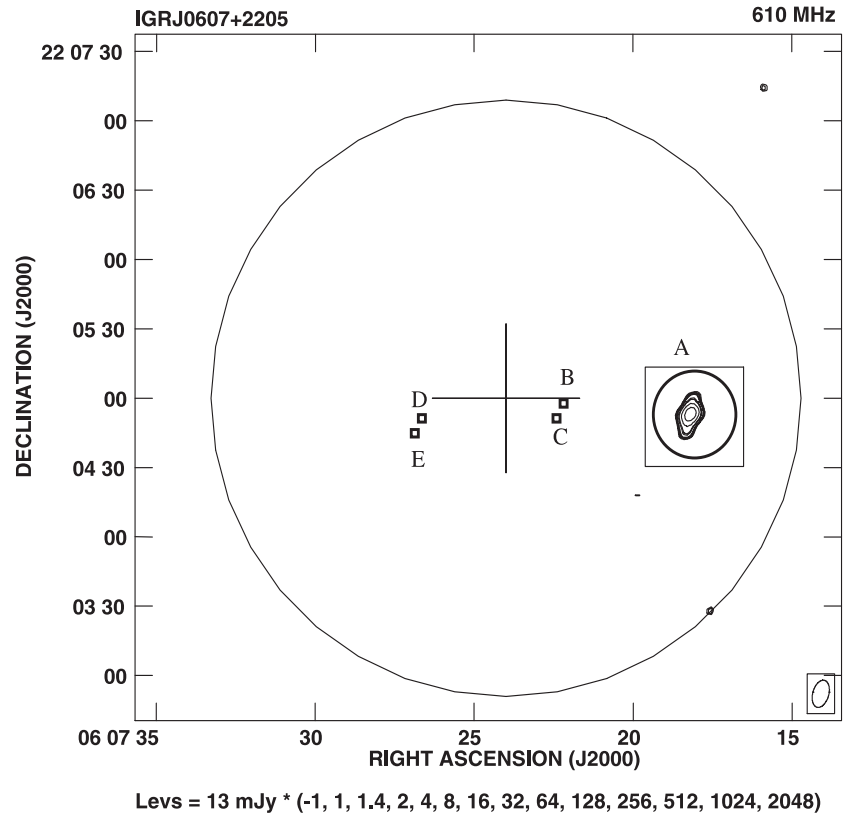

Fig. 5. GMRT image of IGR J06074+2205 at $0.61 \mathrm{GHz}$ with the X-ray source position in the center marked with + . The large circle represents the INTEGRAL uncertainty error circle of $1.6 \sigma$. The bold circle marked with "A" shows the radio source detected by GMRT. The box indicates the known NED field source, A: NVSS J060718+220452, B: DSS J060722+220500, C: 2MASS J060722+220453, D: DSS J060727+220453 and E: 2MASS J060727+220445.

The hardness ratio derived from the RXTE/ASM light curve suggests that the source was in a high state during our observations. The EGRET source 3EG J0617+2238 lies close to the $\mathrm{X}$-ray source; however, it is likely to be associated with the SNR IC443 (Torres et al. 2003). GMRT observations for the source were made on four occasions during Feb. - May 2004 and a radio source was discovered within the INTEGRAL position uncertainty limit of $1.6 \sigma\left(\sim 2^{\prime}\right)$.

Figure 5 shows the GMRT radio image of the IGR J06074+2205 field at $0.61 \mathrm{GHz}$ with a source with a flux density level of $\sim 80 \mathrm{mJy}$ close to the X-ray source position (offset of $1.29^{\prime}$ with respect to the INTEGRAL position). The compact nature of the radio source is inferred from the Gaussian fit to the source. The GMRT position coordinates are (J2000) RA: 06h 07m 18.45s and Dec: +22d 04' 52.49". The source was also detected at $1.28 \mathrm{GHz}$ by GMRT on 1st Feb. 2004 at a flux density of $\sim 36 \mathrm{mJy}$. An observation of this region with the Ryle Telescope at $15 \mathrm{GHz}$ on 29th Jan. 2004, 3 days before our observations, showed an unresolved flux density of $4.0 \pm 0.2 \mathrm{mJy}$, at the NVSS J2000 position (Pooley 2004). The positive detection of the source at different epochs with GMRT at $0.61 \mathrm{GHz}$ clearly suggests a persistent nature for the source in the radio region. The source has shown small variability in the radio flux density.

In Fig. 6, we plot the radio spectrum of the source using all available data. It is seen from the figure that the data can be represented by a power-law of the form $\left(S_{v} \sim v^{\alpha}\right)$. The spectral index at higher frequencies is derived as $-0.70 \pm 0.08$. A power-law nature of the radio spectrum is typical for a 


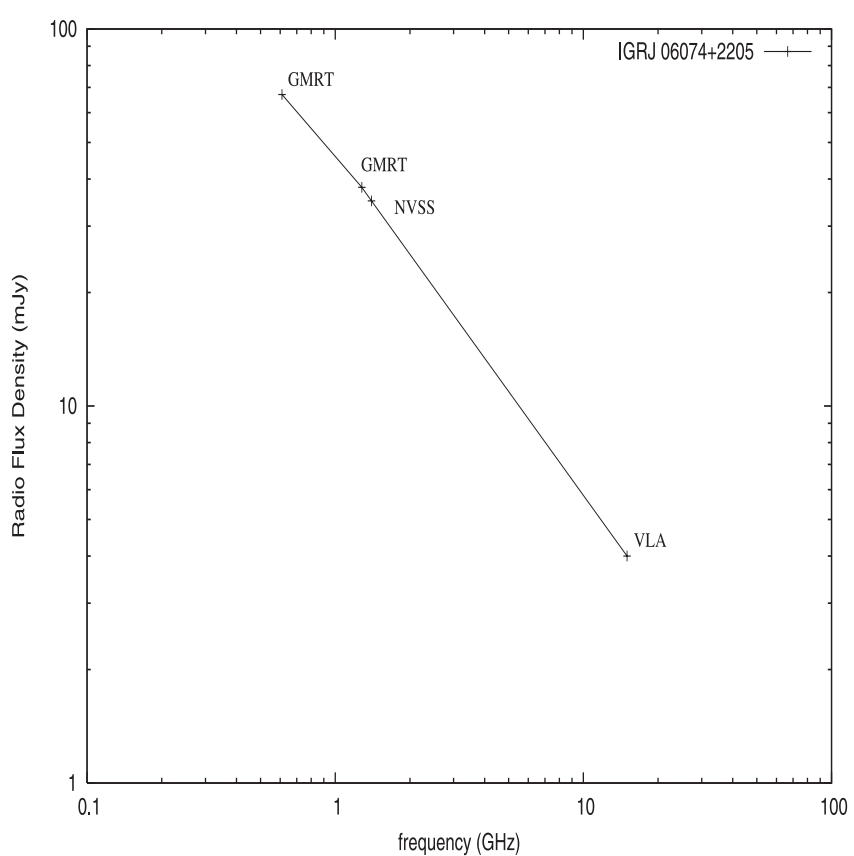

Fig. 6. Radio spectrum for IGR J06074+2205.

non-thermal emission arising from an optically thin medium similar to that produced in the jets interacting with the ambient medium, as seen in extragalactic sources. The hypothesis of a continuous jet can also lead to X-ray emission in the hard $\mathrm{X}$-ray band due to inverse Comptonization of thermal disk photons by a corona (e.g. Markoff $\&$ Nowak 2004). Figure 5 also shows infrared (2MASS J060727+220453, C and 2MASS J060727+220445, E) and optical (DSS J060727+220453, D and DSS J060722+220500, B) sources present in the $1.6 \sigma$ error circle lying close to the $\mathrm{X}$-ray source and thus showing no possible association between the radio and the infrared/optical sources.

IGR J15479-4529: this source was discovered by INTEGRAL in Feb., 2003 observations of Black Hole Candidates (BHC) $4 \mathrm{U}$ 1630-47 at a flux density level of $\sim 3 \mathrm{mCrab}$ between 20-40 keV (Tomsick et al. 2004). An X-ray source 1RXS J154814.5-452845, which lies at the distance of $3^{\prime}$ from IGR J15479-4529 has been detected during XMM-Newton observation of the source field in the energy range below $10 \mathrm{keV}$ (Haberl et al. 2002).

During the GMRT observation, a radio source with a total flux density of $22.2 \mathrm{mJy}$ was detected within the position error box of $3 \sigma$ of the hard X-ray source and is shown in Fig. 7. The GMRT position of the source is (J2000), RA: $15 \mathrm{~h} 47 \mathrm{~m} 44.26 \mathrm{~s}$ and Dec: $-45 \mathrm{~d} 32^{\prime} 35.22^{\prime \prime}$. The rms noise in the GMRT data was $0.5 \mathrm{mJy} \mathrm{b}^{-1}$ in the source direction. The position offset with respect to the INTEGRAL position is $3.98^{\prime}$. The radio morphology of the source suggests that the source is extended in nature. No NVSS image is available for comparison to our measurement. In the absence of RXTE/ASM light curve, it is difficult to comment whether the X-ray source was in the high soft or low hard state during our measurements.

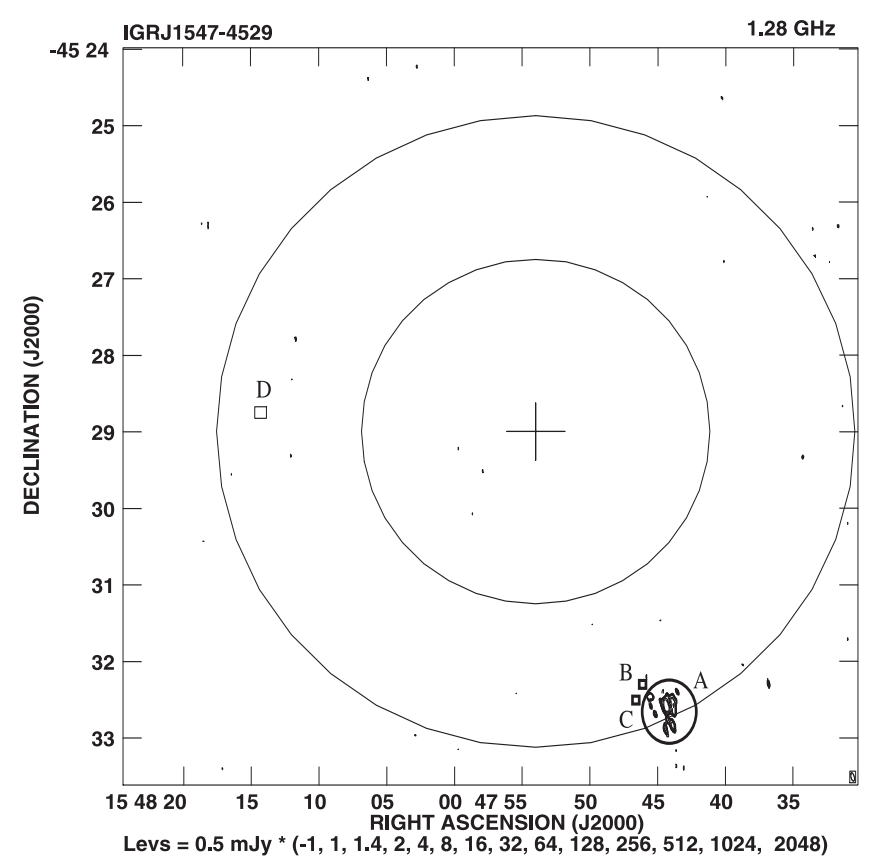

Fig. 7. GMRT image of IGR J15479-4529 at $1.28 \mathrm{GHz}$ with the INTEGRAL position marked with + in the centre. The small and large circles represent the INTEGRAL position uncertainty error circles of $1.6 \sigma$ and $3 \sigma$. The bold circle marked with "A" shows the radio source detected by GMRT. The boxes show the known NED field sources, B: DSS J1547-4532, C: 2MASS J1547-4532 and D: IRXS J154814.5-452845.

It is seen from the figure that the position coordinates of the radio source do not coincide with those of IRXS J154814.5-452845 (D) but they coincide with those of the infrared (2MASS J1547-4532, C) and optical (DSS J1547-4532, B) sources in position, thus suggesting a possible association of the radio and optical/infrared sources. From the above information we conclude that the radio source is not associated with the X-ray source. Since the X-ray counterpart is expected to fall in $90 \%$ of the INTEGRAL position error circle, i.e., within a distance of $1.6 \sigma$, this X-ray source may be a possible candidate for further observations to look for counterparts.

IGR J16479-4514: is a weak hard X-ray transient source with an observed flux density of $\sim 8 \mathrm{mCrab}$ between $25-50 \mathrm{keV}$ (Molkov et al. 2003). The EGRET source 3EG J1655-4554 (Romero et al. 1999) is located close to the X-ray source. The RXTE/ASM hardness ratio suggests that the source was in a high soft state during our observations. The ASM light curve for the source shows that the X-ray flux density between 1st Jan. and 28th Feb. 2003 was relatively constant, though irregular large random spikes were seen.

During our observations with GMRT in Feb. 2004, a bright radio source was detected within the INTEGRAL position uncertainty limit of $1.6 \sigma$. The flux density recorded at $1.28 \mathrm{GHz}$ is $\sim 2.50 \mathrm{Jy}$ with the rms noise of $\sim 1.24 \mathrm{mJy}$. Figure 9 shows the $10^{\prime}$ image of the field of IGR J16479-4514.

The position offset is $3.30^{\prime}$ with respect to the $\mathrm{X}$-ray source position. The radio position of the source is $(\mathbf{J} 2000)$ 


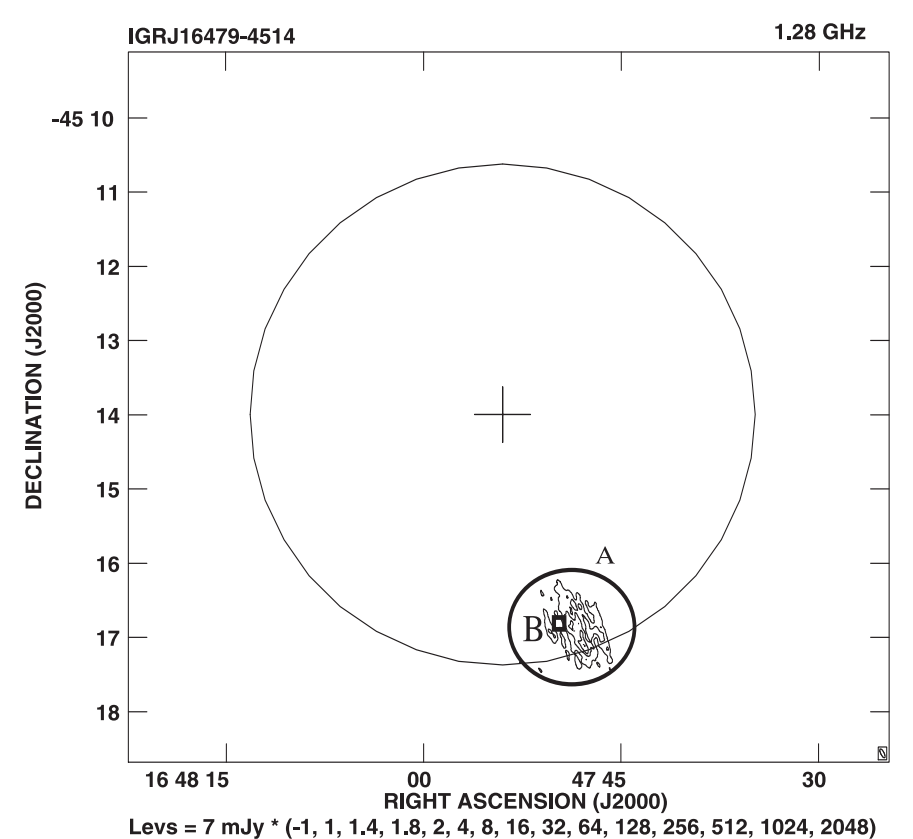

Fig. 8. GMRT image of IGR J16479-4514 at $1.28 \mathrm{GHz}$ with the INTEGRAL position marked with +. The circle shows the INTEGRAL uncertainty error circles of $1.6 \sigma$. The bold circle marked with "A" shows the radio source detected by GMRT and the box shows the known field source, B: 2MASS J164745-451653.

RA: $16 \mathrm{~h} 47 \mathrm{~m} 47.75 \mathrm{~s}$ and Dec: $-45 \mathrm{~d} 17^{\prime} 07^{\prime \prime}$. There is no NVSS image of the field. Radio morphology of the source suggests it is an HII region. While the photoelectric effect heats the gas in the compact HII region leading to ionized hydrogen, the dominant cooling process is mainly through recombination giving rise to free-free radio emission. Thus radio emission from this source should be thermal in nature. The infrared source 2MASS J164745-451653 (B) coincides in position with the radio source. Due to the extended nature of the radio source we conclude from our observations that the radio and infrared sources are not associated with the Galactic X-ray source.

IGR J21247+5058: this source was discovered in the Norma arm region (Walter et al. 2004). The X-ray source has been identified with the core of a bright radio galaxy, 4C 50.55, also known as GPSR 93.319+0.394, KR2, NRAO 659 or BG 2122+50 (Ribo et al. 2004; Combi et al. 2005) and is also coincident with the IR source 2MASS J21243932+5058259. The optical spectrum of the source is very peculiar, having a broad hump around $6700 \AA \mathrm{H} \alpha$ line typical of elliptical or spiral bulge (Barth et al. 2001). While the observed $\mathrm{Na}, \mathrm{Ca}$ and $\mathrm{Mg}$ features are consistent with redshift $z=0$, the identification of the $\mathrm{H} \alpha$ feature leads to a redshift $z=0.020 \pm 0.001$. In addition, the photometric magnitudes indicate an increased reddening from $R$ to the near infrared band. Masetti et al. (2004) therefore propose that the observed spectral features in the optical band are caused by the chance alignment of a F-type star with the radio galaxy. The radio observations of the region with GMRT were carried out on 4 occasions between Apr.-May 2004. The observation details are listed in Table 2. A radio flux

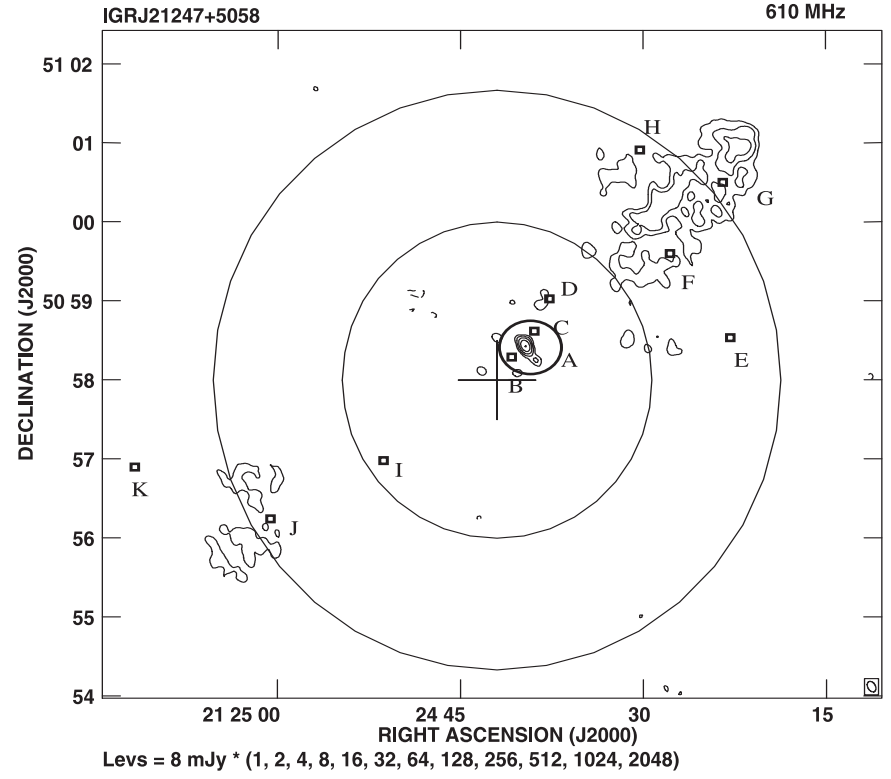

Fig. 9. A high resolution GMRT image of IGR J21247+5058 at $0.61 \mathrm{GHz}$. The core is marked with "A". The X-ray position is marked with + . The boxes show the known field sources, B: 2MASS J2124+5058, C: NVSS 21247+5058, D: 28P206, E: MG4 J2124+5058, F: *87GB 2122+5046, G: 7C 2122+5047, H: NRAO 0659, I: *87GB $2123+5044, \mathrm{~J}: * 87 \mathrm{~GB} 2123+5043, \mathrm{~K}$ : MG4 J212511+5056.

density of $\sim 180 \mathrm{mJy}$ at $0.61 \mathrm{GHz}$ from the core was observed on each occasion (Table 2 ).

In Fig. 9 we have plotted the high resolution GMRT image of the source along with the other radio sources detected in this region in previous surveys. It is seen from the figure that morphology of the GMRT radio image resembles that of a radio galaxy with the bright core marked " $\mathrm{A}$ " and extended radio lobes. However, the field is very complex and contains multiple objects within the $3 \sigma$ position error circle of the INTEGRAL source, and some of them are not seen in the GMRT data. Apart from the GMRT source marked "A", radio sources 28P206 (D) and $87 \mathrm{~GB} 212310.9$ (I) lie within the $1.6 \sigma$ position error circle of the X-ray source. However, the alignment of these sources towards the radio lobes clearly suggests that sources (D) and (I) represent regions of radio lobes that were brighter during the respective observations.

The observed GMRT flux density for the radio core varied between $173 \mathrm{mJy}$ and $185 \mathrm{mJy}$ at $0.61 \mathrm{GHz}$ during our four observations the total flux densities for the radio lobes was measured to be $\sim 1.8 \mathrm{Jy}$ and $\sim 0.85 \mathrm{Jy}$. Using NVSS flux density values for the core and the extended radio lobes, the spectrum for the extended region was fit with a power law spectrum $\left(S_{v} \propto v^{\alpha}\right)$ with a spectral index of $\alpha \sim-0.6$ which is consistent with the synchrotron emission from a optically thin medium. The spectral index of the radio core is $\alpha \sim 0.5$, which clearly points towards an inverted spectrum due to strong absorption in the optically thick medium at low frequencies. Our observation at low frequency $(0.61 \mathrm{GHz})$ with GMRT is indicative of the true spectral nature of the core of IGR J21247+5058. The discovery of the X-ray source in the hard X-ray band, the $\mathrm{H} \alpha$ hump in the optical spectrum and its spectral nature in the radio 
and X-ray bands suggest that IGR J21247-5058 is a partially obscured extragalactic X-ray source similar to NGC1068 and NGC4579.

\subsection{Sources with no radio detection}

IGR J16316-4028: this source was discovered at a flux density level of $\sim 25 \mathrm{mCrab}$ between $20-40 \mathrm{keV}$ during the INTEGRAL survey (Rodriguez \& Goldwurm 2003). The X-ray source is transient in nature, and variability of the X-ray flux density on timescales of $\sim 2000 \mathrm{~s}$ has been observed from the source along with the flaring events during its discovery (Rodriguez et al. 2003). An EGRET gamma ray source 3EG 1631-4033 lies in its direction at a distance of $10^{\prime}$ with respect to the source, IGR J16316-4028 (Rodriguez et al. 2003) suggesting a possible association of the two due to the large position uncertainty of the gamma ray source. During our observations with GMRT at $0.61 \mathrm{GHz}$ and $1.28 \mathrm{GHz}$, no radio source was detected within the X-ray position error box of $3 \sigma$ and upper limits of $\sim 0.8 \mathrm{mJy} \mathrm{b}^{-1}$ and $\sim 6 \mathrm{mJy} \mathrm{b}^{-1}$ were measured.

IGR J16318-4848: this transient source was discovered by INTEGRAL in the Norma arm. The observed flux density in the $15-40 \mathrm{keV}$ band was $50-100 \mathrm{mCrab}$. The X-ray flux data show significant temporal variations on a time scale of $1000 \mathrm{~s}$. A bright 9th mag infrared source in the $J, H, K$ band, coincident with the X-ray source position is seen in the 2MASS and Mid course Space Experiment (MSX) data. The source is highly absorbed at 8 micron flux density and is Galactic in nature (Courvoisier et al. 2003). The best fit position (J2000) determined from the infrared and the XMM measurement corre-

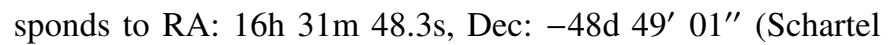
et al. 2003; de Plaa et al. 2003; Revnivtsev et al. 2003). The infrared and optical observations of the counterpart have revealed the high mass X-ray binary nature of the source, there being a compact companion around a supergiant primary $\mathrm{B}[\mathrm{e}]$ star (Filliatre et al. 2004). Strong photo absorption and the fluorescent emission line of neutral iron $(6.4 \mathrm{keV})$ are seen in the XMM data thereby suggesting accretion due to a stellar wind (Matt et al. 2003). A search for the radio emission from the source at 4.8 and $8.6 \mathrm{GHz}$ was made by Walter et al. (2004) with ATCA, but no significant radio flux density was detected from the source with a $1 \sigma$ upper limit of $0.1 \mathrm{mJy}$. We made repeated observations at $0.61 \mathrm{GHz}$ using GMRT at various epochs during 2003 and 2004 observing cycles. No positive detection was made in any of these observations. The upper limit of $\sim 7 \mathrm{mJy}$ for the radio emission within the $3 \sigma$ position uncertainty circle is derived. The typical rms noise in the field is measured to be $\sim 2.2 \mathrm{mJy}$. The non detection at radio frequencies may be due to the highly absorbed nature of the source due to its location in the Galactic plane. The present observation may also correspond to the high soft state of the source during which the radio emission is believed to be quenched. There is also the possibility that the radio jet is not present in the source, as in the case of pulsars.
IGR J16320-4751: the variable hard X-ray source, IGR J16320-4751, was discovered in Feb. 2003 (Tomsick et al. 2003). The hard X-ray source position is consistent with that of the source AX J1631.9-4752. The ASCA/GIS observations also suggest a variable nature of the source (Sugizaki et al. 2001a) and its energy spectrum fits an absorbed power law and so it is believed to be a high-mass X-ray binary system. The variations of the spectral index in different observations by XMM-Newton, ASCA and BeppoSAX suggest that the source undergoes a spectral transition similar to those in the other XRBs. The EGRET source 3EG J1639-4702 (Romero et al. 1999) could be associated with the X-ray source considering the large position uncertainties. The infrared images from the 2MASS database and the optical observations indicate a bright and massive companion for the X-ray source (Rodriguez et al. 2003).

GMRT observation of the field did not show any significant radio source within the $3 \sigma$ position uncertainty limit during different observations at various frequencies. The $3 \sigma$ upper limit for the radio flux density was $\sim 3 \mathrm{mJy}$ at $1.28 \mathrm{GHz}$ with a rms noise of $\sim 0.83 \mathrm{mJy} \mathrm{b}^{-1}$, while the $3 \sigma$ upper limit at $0.61 \mathrm{GHz}$ corresponds to $4 \mathrm{mJy}$ with $\mathrm{rms}$ noise typically $\sim 1.04 \mathrm{mJy} \mathrm{b}^{-1}$. No NVSS data are available for comparison of the field and there is no RXTE/ASM data to determine the X-ray state of the source during our observation. The non-detection of the source at low frequency suggests out either an absorption of the jet in the ambient medium or the lack of a radio jet.

IGR J16418-4532: this hard X-ray source was discovered in the IBIS/ISGRI data at the flux density level of $\sim 3 \mathrm{mCrab}$ in the 20-40 keV bands (Tomsick et al. 2004). The X-ray source is located in the Norma arm region. The GMRT observations at $0.61 \mathrm{GHz}$ were made in Feb. 2004, to search for the radio counterpart of the X-ray source. No significant radio flux density was detected within the INTEGRAL position uncertainty limit of $2^{\prime}$. The $3 \sigma$ upper limit is $1 \mathrm{mJy}$ at $1.28 \mathrm{GHz}$ with a rms noise of $\sim 0.33 \mathrm{mJy} \mathrm{b}^{-1}$. No RXTE/ASM X-ray light curve or the NVSS image is available. Thus suggesting the absence of the radio emission from the X-ray source during our observations.

IGR J17391-3021: hard X-ray emission in the $18-50 \mathrm{keV}$ bands from the transient HMXB source, IGR J17391-3021, was discovered in Aug. 2003 at a flux density level of $\sim 70$ mCrab (Sunyaev et al. 2003a). The position coordinates of the source overlap with the X-ray source XTE J1739-302 with J2000 position of, RA: $17 \mathrm{~h} \mathrm{39m} \mathrm{11.1s} \mathrm{and} \mathrm{Dec:}-30 \mathrm{~d} 20^{\prime} 37^{\prime \prime}$. Using CHANDRA observations, the position has been refined to $1^{\prime \prime}$. Within the improved position, an optical counterpart of the source is identified with the object 052528760590 in the USNO A2 catalog. It is also a 2MASS source and is a highly reddened O star (Smith et al. 2003). A radio source was detected in a VLA observations at $4.9 \mathrm{GHz}$ at a flux density of $0.9 \pm 0.2 \mathrm{mJy}$, and a J2000 position of, RA: $17 \mathrm{~h} 39 \mathrm{~m} 01.52 \mathrm{~s}$ and Dec: $-30 \mathrm{~d} 19^{\prime} 34.9^{\prime \prime}$ within the $3^{\prime}$ error circle INTEGRAL position but well outside the CHANDRA error circle 
(Rupen et al. 2003b). The EGRET source 3EG J1736-2908 is located near the X-ray source.

The source was observed with GMRT at $1.28 \mathrm{GHz}$ and no radio emission was detected from the field within a $3 \sigma$ position uncertainty of $2^{\prime \prime}$, as determined from CHANDRA data. The $3 \sigma$ upper limit for the radio flux density from GMRT data is $\leq 1 \mathrm{mJy}$ and the rms noise was $0.2 \mathrm{mJy} \mathrm{b}^{-1}$. No significant radio flux from the X-ray source position was detected in the analysis of the NVSS data and the $3 \sigma$ upper limit for the radio flux density is derived as $2.5 \mathrm{mJy}$.

Nevertheless, during our observations, we did detect a radio source coincident in position with the NVSS source with radio flux density of $8.9 \mathrm{mJy}$ and rms noise of $0.19 \mathrm{mJy} \mathrm{b}^{-1}$ at J2000 position of, RA: $17 \mathrm{~h} 39 \mathrm{~m} \mathrm{06s}$ and Dec: $-30 \mathrm{~d} 18^{\prime} 12^{\prime \prime}$ which lies $2.4^{\prime}$ away from the X-ray source position. Thus we confirm from the above data that the radio source detected by VLA in the field of the X-ray source is not associated with the Galactic X-ray source.

IGR J17544-2619: this transient source was discovered with INTEGRAL at a flux density of $\sim 160 \mathrm{mCrab}$ in the 18 $25 \mathrm{keV}$ bands (Sunyaev et al. 2003b). The X-ray variability with time scales of $1.5-2 \mathrm{~h}$ seen in the data is ascribed to the binary nature of the X-ray source and is associated with the accretion-emission processes (Rodriguez et al. 2003). A ROSAT source IRXS J175428.3-262035 with the count rate of $0.08 \pm 0.02$ counts s$^{-1}(0.1-2.4 \mathrm{keV})$ lies within the field of IGR J17544-2619. Optical and infrared parameters observed in the 2MASS survey give the magnitude $J=8.79 \pm 0.02$, $H=8.31 \pm 0.03, K=8.02 \pm 0.03$, thus suggesting the companion star as an early O-type star.

The GMRT image of the field of source IGR J17544-2619 at $0.61 \mathrm{GHz}$ showed no significant radio flux density within the $3 \sigma$ position uncertainty of the X-ray source. The $3 \sigma$ upper limit for the radio emission from the GMRT data is $<7 \mathrm{mJy}$ with the rms noise of $\sim 2.2 \mathrm{mJy} \mathrm{b}^{-1}$. No radio emission from the source region is seen even in the NVSS image. The $3 \sigma$ upper limit from the NVSS data is estimated to be $\sim 3 \mathrm{mJy}$. The RXTE/ASM hardness ratio data for the source suggests that it was in the high soft state during our observations. The non-detection of radio emission within the $4^{\prime \prime}(1.6 \sigma)$ (Gonzalez-Riestra et al. 2004) position limit from XMMNewton observation, during the high soft X-ray state of the source suggests that there is no associated radio source for the $\mathrm{X}$-ray source; however, the radio emission, if present, may be synchrotron self-absorbed at low frequency or highly variable, similar to some of the HMXBs below $1 \mathrm{GHz}$.

IGR J17597-2201: the X-ray source is a weak transient source with a flux density level of $5 \mathrm{mCrab}$ in the $15-40 \mathrm{keV}$ energy band (Lutovinov et al. 2003b). The RXTE observation shows variability of the source. The PCA spectra is, however, consistent with a power law with an additional soft X-ray excess. The measured spectral indices in the hard X-ray band range between 1.8 and 2.9. An Fe $\mathrm{K}$ emission line at $6.5 \mathrm{keV}$ has been detected (Markwardt et al. 2003) in X-ray. These observations suggest that IGR J17597-2201 is a binary system containing a neutron star as the compact companion. The
GMRT observations on 16th Sep. 2003 at $0.61 \mathrm{GHz}$ were taken immediately after the discovery of the source with INTEGRAL. The radio image of the field showed no significant radio source within the $3 \sigma$ position uncertainty circle of the X-ray source position. A $3 \sigma$ upper limit for the radio flux density is derived as $<7 \mathrm{mJy}$ with the rms noise of $\sim 2.14 \mathrm{mJy} \mathrm{b}^{-1}$. The analysis of the NVSS data also did not yield any radio flux density at $1.4 \mathrm{GHz}$ andthe $3 \sigma$ upper limit is $3.5 \mathrm{mJy}$. An upper limit for the radio emission from the source clearly implies that if the immediate radio emission was indeed present, then either it was highly absorbed at low frequency or was variable.

IGR J18325-0756: this is a transient, flat spectrum X-ray source discovered with IBIS at a flux density level of $\sim 10 \mathrm{mCrab}$ in the $15-40 \mathrm{keV}$ band and $\sim 5 \mathrm{mCrab}$ in the $40-100 \mathrm{keV}$ band. The source is variable in the $\mathrm{X}$-ray range (Lutovinov et al. 2003c). We observed the source with GMRT at $0.61 \mathrm{GHz}$ during $\mathrm{AO} 3$ to look for any residual low frequency flux density from the active phase. No significant radio source was detected within the $3 \sigma$ INTEGRAL position error circle. The GMRT $3 \sigma$ upper limit for the radio flux density is $\leq 3 \mathrm{mJy}$. Thus our conclusion for non-detection of radio emission is similar to IGR J17597-2201.

IGR J18483-0311: this new transient source was discovered in Apr. 2003 with a X-ray flux density of $\sim 10 \mathrm{mCrab}$ in the 15-40 keV band and a flaring behavior in the X-ray light curve (Chernyakova et al. 2003). The radio image of the field of IGR J18483-0311 with GMRT at $1.28 \mathrm{GHz}$ showed no significant radio source within the $3 \sigma$ upper limit of $4 \mathrm{mJy}$ either at $0.61 \mathrm{GHz}$ or $1.28 \mathrm{GHz}$. No significant source was detected in the NVSS data. The $3 \sigma$ upper limit at $1.2 \mathrm{GHz}$ was $1 \mathrm{mJy}$. The non-detection of the radio emission supports our previous conclusion.

IGR J19140+0951: the X-ray source was discovered with IBIS/ISGRI at a flux density of 50-100 mCrab between 15-40 keV (Hannikainen et al. 2003). The RXTE light curve of the source shows variations on short time scales. No QPO was seen in the power density spectrum of the source in the PCA/RXTE data but the X-ray spectrum is well fitted by power-law + thermal component (Cabanac et al. 2004a). A broad ionized iron line is also seen in the spectral data. The source shows strong spectral variability more typical of X-ray binaries. A comparison of the ISGRI/JEMX and RXTE/PCA spectra reveals a state transition in the source from "steep power-law" to "low hard" (Schultz et al. 2004). Such a transition in spectral state is a characteristic of a BH LMXB (McClintock et al. 2003). However; Rodriguez et al. (2005) and in't Zand et al. (2003) confirm the HMXB nature of the source, with a neutron star as the central engine based on available RXTE and INTEGRAL data. We performed a series of ten radio observations with GMRT for the source at 0.61 and $1.28 \mathrm{GHz}$ during the 2003 and 2004 observing seasons. No positive radio detection within the $1.6 \sigma$ position error circle of $1.3^{\prime}$ around the new refined position coordinate (Cabanac et al. 2004b) of the source was found during our observations. The upper limit for the GMRT radio flux density towards the source direction is 
derived as $\leq 5.5 \mathrm{mJy}$. No radio source corresponding to the X-ray source position was seen even in the NVSS data at $1.4 \mathrm{GHz}$. The absence of radio emission from the source as seen in other X-ray binaries, such as XTE J1748-288, GRS $1758-258$ below $1.4 \mathrm{GHz}$ is also seen in IGR J19140+0951. Thus we confirm from our observations that the X-ray source has no radio counterpart, or if present, it is highly absorbed below $1.4 \mathrm{GHz}$.

\section{Discussion}

Among the seventeen sources for which GMRT data is presented in the previous section, radio counterparts are detected for seven objects that lie within the $3 \sigma$ position error circle of the INTEGRAL source positions. Among these seven radio sources, the morphology for IGR J16479-4514 resembles HII region while that of IGR J18539+0727 and IGR J21247+5058 indicates a radio galaxy. In the case of IGR J15479-4529, GMRT data do not fit a point source model, thereby suggesting an extended morphology more likely of an extragalactic object. The sources IGR J06074+2205 and IGR J18027-1455 are unresolved sources with extragalactic properties and are probably AGNs. The INTEGRAL source IGR J17091-3624 for which coincident radio emission is measured from the GMRT data is a binary source and a possible microquasar candidate.

The sources IGR J16318-4848, IGR J16320-4751, IGR J17544-2619, IGR J17597-2201 and IGR J19140+0951 are suggested as X-ray binaries from the INTEGRAL studies; however, their radio counterparts are not yet detected, suggesting that either they are synchrotron self absorbed at low frequencies or have no current radio emission. The sources IGR J16316-4028, IGR J16418-4532, IGR J17391-3021, IGR J18325-0756 and IGR J18483-0311 do not have low frequency radio counterparts and were not identified based on our survey. This points to either the variable nature of the radio emission or a persistent emission at much lower flux densities than the GMRT threshold. As seen in Table 2, the position offset of the possible radio counterparts with respect to the X-ray source position are of the order a few arcminutes and are well within the $3 \sigma$ error circle of the X-ray position. Hence such possible identifications are a reason to conduct even deeper optical and infrared follow-up observations.

A large number of accreting binary systems show a transient behavior in the X-ray band. The radio emission from $\mathrm{X}$-ray binaries can be classified into two broad classes (1) $\mathrm{X}$-ray sources with persistent radio emission that are generally binary systems containing a $\mathrm{BH}$ or a NS with low magnetic field; (2) the sources with a highly variable and bright radio emission, associated with jet-like outflows from their compact objects.

On the basis of X-ray spectral characteristics, their transient nature and their discovery in the high energy band, a majority of the INTEGRAL sources are believed to be Galactic HMXB. It is therefore likely that during the transient outbursts, most of these sources will produce relativistic outflows, thereby giving rise to strong radio emission. Therefore, non-detection of radio flux density from a number of INTEGRAL sources may be due to the different X-ray state of the source at the time of our observations. Thus, near simultaneous observations in different wave bands are necessary to reveal the true nature of most of the INTEGRAL high energy sources.

Acknowledgements. We acknowledge the excellent support provided by the GMRT staff during our observations. We are also grateful to our colleagues at TIFR and NCRA for helpful discussions. This research had made extensive use of the NASA/IPAC extragalactic sources data base NED, Data from the NASA/NVSS survey and the public archives from the RXTE and INTEGRAL Satellites. M.P. is grateful to Director of the NCRA for providing the research facilities in Pune and to Prof. V. H. Kulkarni of Mumbai University for his encouragement. This research is supported by ISRO, grant No. DOS. 9/2/109/2003-II under its RESPOND program. MP also thanks Prof. Kulkarni, and Witta, Green, Rodriguez, and Ribo for useful discussions.

\section{References}

Basko, M., Sunyaev, R., \& Titarchuk, L. 1974, A\&A, 31, 249 Belloni, T., Mendez, M., King, A., et al. 1997, ApJ, 488, L109

Bird, A., Barlow, E., Bassani, L., et al. 2004, ApJ, 607, L33

Barth, A., Luis, H., Filippenko, A., et al. 2001, ApJ, 546, 205

Cabanac, C., Rodriguez, J., Hannikainen, D., et al. 2004a, ATEL, 272

Cabanac, C., Rodriguez, J., Petrucci, P., et al. 2004b, RMxAC, 20, 209

Ceballos, M., \& Barcons, X. 2004, Multiwavelength AGN surveys Conf. Proc., Cozumel, 17

Chenevez, J., Budtz-Jorgensen, C., Lund, N., et al. 2004, ATEL, 223

Chernyakova, M., Lutovinov, A., et al. 2003, ATEL, 157

Combi, J., Ribó, M., \& Mirabel, I. F. 2004, ATEL, 246

Combi, J., Ribó, M., \& Mirabel, I. F. 2005, Proc. Multiwavelength Approach to Unidentified Gamma-Ray Sources, ed. K. S. Cheng, \& G. E. Romero, Ap\&SS, 297, 385

Condon, J. J., Cotton, W. D., Greisen, E., et al. 1998, AJ, 115, 1693

Condon, J. 1999, Proc. Natl. Acad. Sci., 96, 4756

Cordier, B., Goldwurm, A., Laurent, P., et al. 1991, Adv. Sp. Res., 11, 169

Courvoisier, T., Walter, R., Rodriguez, J., et al. 2003, IAUC, 8063

de Plaa, J., Hartog den, P., Kaastra, J., et al. 2003, ATEL, 119

Fenimore, E., \& Cannon, M. 1978, Appl. Opt., 17, 337

Filliatre, \& Chaty 2004, ApJ, 616, 496

Matt, G., \& Guainazzi, et al. 2003, MNRAS, 341, L13

Gonzalez-Riestra, E., Oosterbroek, T., Kuulkers, E., et al. 2004, A\&A, 420, 589

Hannikainen, D., Rodriguez, J., Pottschmidt, K., et al. 2003, IAUC, 8088

Haberl, F., Motch, C., \& Zickgraf, F. 2002, A\&A, 387, 201

Ishwara-Chandra, A. P. Rao, Pandey, M., et al. 2005, ChJAA, 5, 87

Kuulkers, E., Lutovinov, A., Parmar, A., et al. 2003, ATEL, 149

Lebrun, F., Leray, J., Lavocat, P., et al. 2003, A\&A, 411, L141

Lutovinov, A., Rodriguez, J., Produit, N., et al. 2003a, ATEL, 151

Lutovinov, A., Shaw, S., et al. 2003b, ATEL, 154

Lutovinov, A., Walter, R., et al. 2003c, ATEL, 155

Malizia, A., Bassani, L., et al. 2004, ATEL, 227

Markoff, S., \& Nowak, M. 2004, ApJ, 609, 972

Markwardt, Swank J., et al. 2003, ATEL, 156

Masetti, N., Palazzi, E., Bassani, L., et al. 2004, A\&A, 426, L41

McClintock, J., \& Remillard, R. 2003, Compact Stellar X-ray sources (Cam. Univ. Press)

Mirabel, F., Gerard, E., \& Rodríguez, L. 1994, IAU Circ., 5958

Mirabel, F., Harmon, B., Deal, K., et al. 1997, ApJ, 477, L85

Mirabel, F., Marti, J., Chaty, S., et al. 1998, New Aston. Rev., 42, 9-10, 621 
Mirabel, F., \& Rodríguez, L. 1999, ARA\&A, 37, 409

Molkov, S., Mowlavi, N., Goldwurm, A., et al. 2003, ATEL, 176

O’ Dea, C., et al. 1998, ApJ, 488, L109

Pandey, M., Durouchoux, Ph., Manchanda, R., et al. 2004, 5th INTEGRAL workshop proceedings held in Munich, ESA, SP-552, 695

Pooley, G. 2004, ATEL, 226

Revnivtsev, M. 2003, A\&A, 411, 329

Revnivtsev, M., \& Lutovinov, A. 2003, Astron. Lett., 29, 719

Ribo, M., Combi, J., \& Mirabel, I. F. 2004, ATEL, 235

Rodriguez, J. 2003, ATEL, 194

Rodriguez, J., \& Goldwurm, A. 2003, ATEL, 201

Rodriguez, J., Tomsick, J., Foschini, L., et al. 2003, IAUC, 8096

Rodriguez, J., Foschini, L., Goldwurm, A., et al. 2004, ATEL, 253

Rodriguez, J., Cabanac, C., Hannikainen, et al. 2005, A\&A, 432, 235

Romero, A., Gaztanaga, E., Barriga., et al. 1999, A\&A, 348

Rupen, M., Mioduszewski, A., \& Dhawan, V. 2003a, ATEL, 152

Rupen, M., Mioduszewski, A., \& Dhawan, V. 2003b, ATEL, 184

Schartel, N., Ehle, M., Breitfellner, M., et al. 2003, IAUC, 8072
Schultz, J., Hannikainen, D., Vilu, O., et al. 2004, A\&A, 423, L17

Smith, D., Heindl, W., Swank, J., et al. 2003, ATEL, 182

Sugizaki, M., Matsuzaki, K., Kaneda, H., et al. 2001a, AIPC, 599, 959

Sugizaki, M., Matsuzaki, K., Kaneda, H., et al. 2001b, ApJS, 134, 77

Sunyaev, R., Lotovinov, A., et al. 2003a, ATEL, 181

Sunyaev, R., Grebenev, S., Lutovinov, A., et al. 2003b, ATEL, 190

Sunyaev, R., Titarchuk, et al. 1980, A\&A, 86, 121

Swarup, G. 1991, Radio Interferometry techn.: Proc. 131st IAU Coll., Astr. Scty. Pac., 376

Tomsick, J., Lingenfelter, R., et al. 2003, IAUC, 8076

Tomsick, J., Lingenfelter, R., Corbel, S., et al. 2004, ATEL, 224

Torres, D., Romero, G., Dame, T., et al. 2003, Phys. Rep., 382, 303

Ubertini, P., Bazzano, A., Cocchi, M., et al. 1999, Astroph. Lett. Comm., 38, 301

Van der Klis, M., \& Wijnands, R. 1999, ApJ, 514, 939

Voges, W., Aschenbach, B., Boller, Th., et al. 2000, VizieR online Data Catalog, IX/29

http://cdsweb.u-strasbg.fr/viz-bin/Cat?IX/29

Walter, R., Bodaghee, A., et al. 2004, ATEL, 229

in't Zand, J. J. M., Heise, J., et al. 2003, ATEL, 229 